\title{
Design and baseline characteristics of the 10 Small Steps Study: a randomised controlled trial of an intervention to promote healthy behaviour using a lifestyle score and personalised feedback
}

\author{
Sanjoti Parekh ${ }^{1,2^{*}}$, Corneel Vandelanotte ${ }^{3}$, David King ${ }^{4}$ and Frances M Boyle ${ }^{5}$
}

\begin{abstract}
Background: Non-communicable diseases (NCDs) are the leading causes of death globally and are associated with a limited set of common, modifiable health behaviours: tobacco use, physical inactivity, harmful use of alcohol and unhealthy diet. General practice offers an ideal avenue for addressing such health behaviours on a population-wide basis. This paper describes the protocol of a multiple health behaviour change intervention designed for implementation in general practice and summarises the baseline characteristics of its participants.

Method/Design: The 10 Small Steps (10SS) study, a randomised controlled trial, involved 4,678 adult general practice patients in Queensland, Australia. Self-reported data were collected to establish the proportion of participants meeting recommended guidelines for ten health behaviours: physical activity, body mass index, alcohol, smoking and six dietary behaviours. Participants were randomised to four groups: contact at baseline only ('single intervention' and corresponding control group) and contact at baseline and 3 months ('dual intervention' and corresponding control group). At each contact the participants received a computer-tailored feedback and one page information sheet according to their allocation to intervention or control groups. Change in the intervention group compared to the control group was assessed at 3 and12 months after baseline data collection.

Responses were summed to calculate an individual lifestyle score (the Prudence Score), which ranged from 0 to 10. The baseline response was $56.5 \%$ (4678 of 8343 invited participants) and the study sample was primarily female (68.7\%) with an average age of 47 years. The mean Prudence Score was 5.8 (95\%Cl 5.75-5.85).

Discussion: Baseline data from the 10SS study show that nearly all participants engage in some health behaviours but relatively few adhere simultaneously to a core set of dietary and lifestyle behaviours associated with risk of NCDs. Ample scope exists to improve health behaviour to reduce NCDs in the general practice setting and the $10 S S$ study trial will provide data on the extent to which a minimal computer-tailored intervention can meet this objective. The protocol developed for the 10SS study has potential for translation into routine general practice as it has minimal impact on practice routine whilst contributing to primary prevention objectives.

Trial Registration: The Australian New Zealand Clinical Trials Registry ACTRN12611001213932

Keywords: Lifestyle score, Non-communicable diseases, Prevalence, Prevention, General practice, Health behaviours, Intervention
\end{abstract}

\footnotetext{
* Correspondence: s.parekh@uq.edu.au

'School of Population Health, The University of Queensland, Herston Road,

Herston, Queensland 4006, Australia

Full list of author information is available at the end of the article
} 


\section{Background}

Non-communicable diseases (NCDs) are a major cause of disability and reduced quality of life. The cost of preventable chronic conditions on health care resources and loss of workplace productivity is escalating. Over the last decade there has been a worldwide increased focus on prevention of NCDs [1,2]. Chronic conditions such as cardiovascular disease, type-2 diabetes, obesity and several cancers are associated to varying degrees with a limited set of common, modifiable health behaviours [3-6]. Globally at least 4.9 million people die each year as a result of tobacco use, 2.6 million as a result of being overweight or obese, 4.4 million as a result of raised total cholesterol levels and 7.1 million as a result of raised blood pressure [7]. The total burden of disease attributed to modifiable risk factors such as high blood pressure (7.6\%), tobacco (7.8\%), alcohol (2.3\%), high cholesterol $(6.2 \%)$, overweight (7.5\%), low fruit and vegetable intake $(2.1 \%)$ and physical inactivity (6.6\%) is high [8]. Adoption of a healthy lifestyle has the potential to significantly reduce morbidity and mortality [9-13].

Most primary health care in Australia is provided within general practice clinics and $86 \%$ of the population visit a general practitioner (GP) each year [14]. GPs have substantial knowledge of population health and are in an ideal position to advise individual patients about lifestyle choices. Therefore the general practice setting offers the potential to facilitate healthy changes in lifestyle on a large scale $[15,16]$. Behavioural approaches to both single and multiple risk factors have been applied in general practice and the evidence suggests that brief interventions can be effective $[17,18]$. Multiple risk factor interventions have been demonstrated to improve health especially in high risk patients. However, there are known barriers to addressing behavioural risk factors in primary care. These include low self-efficacy of GPs for providing health behaviour change information, competing pressures on time, lack of supportive organisational infrastructure or funding to support assessments $[19,20]$. Overall, the available evidence indicates the need for lifestyle risk factor interventions that can be incorporated into routine care with minimal disruption to general practice activities and limited demands on GPs' time [21,22].

The primary aim of this paper is to describe the protocol for the 10SS study, which was designed to evaluate the use of personalised computer-generated health promotion advice to address multiple health behaviours in the general practice setting. The secondary aim is to describe baseline characteristics of the study participants, including the prevalence and patterns of multiple risk behaviours and their socio-demographic correlates.

\section{Methods/Design}

\section{Study design and sampling}

The 10 SS study design was a $2 \times 2$ randomised controlled trial. The study protocol was designed to answer the specific question: 'Can a low intensity computer-tailored intervention implemented through general practice be used to motivate large numbers of individuals to adopt and maintain a healthier lifestyle and diet?' There were specifically two parts to this study aim: (i) develop and test an intervention designed to produce short-term (at 3 months after baseline) improvement in ten health behaviours; and (ii) test if a second intervention contact at 3 months improves maintenance of health behaviour change over and above a single one-off intervention at 12 months.

Thirty general practitioners, from metropolitan area in Brisbane, Australia, were invited to participate in the study in 2008. In each participating general practice the practice manager generated a list of potential participants - patients aged between 18 and 70 years who had visited the practice in the preceding six months and had no apparent active cancer, ongoing need for dialysis, recent cardiovascular event, dementia, other terminal illness or recent bereavement. After the treating GP had vetted the list of potential participants to confirm eligibility each patient received a written invitation to take part in the study together with a reply-paid questionnaire. All letters to patients used the corresponding GP's letterhead and included the doctor's electronic signature.

To increase participation in the study non-respondents were sent up to two reminder letters and a new copy of the questionnaire at intervals of three weeks. Completion and return of the questionnaire was regarded as consent to participate in the project. Patients that declined to participate at any stage were subsequently excluded from the study. The data were collected between August and October 2008. Ethics approval for the study was granted by the Behavioural and Social Sciences Ethical Review Committee of the University of Queensland.

\section{Sample size calculations}

The sample size for recruiting the participants was calculated based on the results of the pilot study which is described elsewhere [23]. Using two-sided $\alpha$ (0.05), to have a 95\% chance of seeing the proportion scoring 6 or more increase to $45 \%$ required 1,756 participants in each group (3,512 overall). A total of 4,220 participants were required to allow for loss to follow-up, and the study therefore aimed to invite 330 patients from each of 20 general practitioners. 


\section{Randomisation}

Using each GP as a unit for randomisation, patients who responded at baseline were randomised using a permuted block randomisation procedure. Participants living at the same residential address were randomised into the same group. Study participants were randomised into intervention and control groups and were further randomised into four groups: receiving the intervention at baseline only ('single intervention') and corresponding control group; and receiving the intervention at baseline and 3 months ('dual intervention') and corresponding control group. Participants in the 'single intervention' had study measures assessed at baseline and 12 months, those in the 'dual intervention' were assessed at baseline, 3 and 12 months.

\section{Intervention}

Participants in the intervention group received individualised feedback regarding their lifestyle score described in Measures section), and additional health promotion material on behaviours for which they failed to meet guideline recommendations, either once ('single intervention') or twice ('dual intervention'). The health promotion material consisted of concise printed information and links to electronic sites for more extensive information and support. The feedback letter encouraged the adoption of at least one additional health behaviour to those already being adhered to.

\section{Control}

The control group received information about health protective behaviours not included in the Prudence Score (sun protection, updating tetanus vaccination, mammogram and Pap smear). Feedback to the control group was provided in an attempt to reduce attrition.

\section{Measures}

Data were collected from patients via a self-administered questionnaire. This questionnaire was previously piloted in two general practices in Brisbane and was found to be valid (as judged by independent corroboration by an individual's spouse or partner) and reliable (test-retest over an interval of three months) [23]. The questionnaire included 26 items related to modifiable health behaviours and eight related to participants demographics. Demographics included age, gender, education, height, weight, postcode, work status and marital status. The dietary factors assessed included consumption of fish, meat, fruits and vegetables $(\mathrm{F} \& \mathrm{~V})$, milk, spread and salt. Data were collected on current and past smoking status using the established questions from the Australian National Health Survey. The number of alcoholic drinks consumed each day of the week was collected to calculate total alcohol intake. Physical activity questions were taken from the International Physical Activity Questionnaire (IPAQ - Short Form), a valid and reliable measure [24]. Body Mass Index (BMI) (kilograms/meter ${ }^{2}$ ) was calculated using respondents' self-reported height and weight.

Guidelines promulgated by the National Health and Medical Research Council of Australia (NHMRC) and the National Heart Foundation of Australia (NHF) were used to determine achievement of behavioural recommendations. The guidelines recommend intake of no more than four serves of meat per week [25]; consumption of at least one serve of fish per week [26]; use of reduced or low fat milk [26]; adding no salt during or after cooking [27]; intake of at least five serves of vegetables and two serves of fruit per day [25]; using margarine or other spreads instead of butter [26]; undertaking at least 150 minutes physical activity per week [28]; consumption of two or less standard drinks of alcohol per day with no binge drinking [29]; being a non-smoker [30] and having body mass index between 18.49 and $24.99 \mathrm{~kg} / \mathrm{m}^{2}$ [31].

Responses to the ten behavioural items were dichotomized as meeting ( 1 point) or not meeting ( 0 point) the criteria and summed to produce a composite lifestyle score, the Prudence Score. The score ranged between 0 and 10. The Prudence Score was used as the main outcome variable. Independent variables were gender (female/ male); age group (18 to 39 yrs/40 to 59 yrs/60 and above); marital status (married or living as married/single, divorced, widowed, never married); employment status (working full or part time/not working due to health, retirement, home duties) and education (less than high school/high school/diploma or trade certificate/university).

Socio-economic Index for Areas (SEIFA) categories were applied as an area level indicator of socioeconomic status and were based on the postcodes provided for each patient. SEIFA ranks areas according to socioeconomic and positional disadvantage based on information derived from the five-yearly Census of Population and Housing in Australia, [32] and is the most widely used general measure of socio-economic status (SES) by area in Australia. The SEIFA sub-code for 'Economic Resources', which includes variables such as income, housing expenditure and assets of households, was used for this analysis. Ten categories of SEIFA were merged into three categories: advantaged, moderately disadvantaged and disadvantaged.

\section{Data management and statistical analysis}

After data entry, data was manually and statistically checked as a part of the data-cleaning process. Initially, descriptive statistics were calculated for all variables. To identify patterns of health behaviours in the study population, the Prudence Score was stratified by demographic characteristics of gender, age, education, marital and 
employment status. A bivariate analysis using the Chi2 test was performed to assess differences between meeting health behaviour recomm and the demographic variables of gender, education, work status, marital status, and area of residence. A two-sample $t$-test was used to assess the differences in the mean Prudence Score for gender, age, education, employment status, marital status and SEIFA.

The Prudence Score was further divided into 3 categories: low (0 to 5$)$, medium ( $6 \& 7$ ) and high ( 8 to 10$)$ as the primary outcome variable in the multivariate analysis. The multivariate logistic model was used to further assess the impact of each demographic factor on the combined score as well as determine characteristics of individuals at highest risk of having unhealthy lifestyles. Results of this model are reported as relative risk ratios. Records for missing values for any of the prudent variables were deleted from the total Prudence Score calculations. The statistical package STATA version 11 (Stata Corporation, 2008) was used for the analysis.

\section{Response rate}

Of the 30 GPs approached, 25 showed initial interest. Four GPs subsequently withdrew because of leave planned during the recruitment period or inadequate computer systems to identify potentially eligible patients. Practice staff identified a total of 8,281 (32\% men) potentially eligible patients for the remaining 21 GPs, of whom 4,678 completed and returned the questionnaire. As such, the participation rate at baseline was $59.9 \%$, after notified deaths and returns to sender were omitted (5.2\%; $\mathrm{n}=412$ ). Figure 1 presents a study flow chart.

\section{Baseline characteristics of the sample}

Baseline socio-demographic characteristics for intervention and control groups are presented in Table 1 . The average age of participating patients was 47 years and the majority were female (68.7\%), married or living as married $(68.8 \%)$, and with a diploma or university degree (56.6\%).

\section{Distribution of health behaviours}

Table 1 summarizes the distribution of the dietary and lifestyle behaviours for all the participants. Most (86\%) were non-smokers whereas only $12.4 \%$ consumed 7 or more serves of vegetables and fruits daily. These ten behaviours are further stratified by gender and age (Tables not included). Women more soften reported adhering to several behaviours than men: eating 4 or less serves of meat per week $(74.6 \%$ versus $57.2 \%$, p < $0.05)$, drinking low fat milk $(72.7 \%$ versus $61.8 \%$, p < $0.05)$ and drinking alcohol within recommended limits (74.0\% versus $56.6 \%, \mathrm{p}<0.05)$. For $46.4 \%$ of women BMI was within the recommended range, as compared to $29.6 \%$ of men. Older patients reported significant higher adherence to recommended behaviours, except for BMI where older patients had lower adherence. No age specific differences were noted for physical activity and salt intake. A significantly lower percentage of married patients had BMI within normal range when compared to patients who were single, widowed or divorced $(40.9 \%$ versus $50.5 \%, \mathrm{p}<0.05)$.

\section{Combinations of health behaviours}

The data were analyzed to assess the proportion of participants that adhered to certain combinations of multiple health behaviours. Only $30 \%$ of patients reported they were non-smokers and adhered to physical activity and alcohol guidelines (see Table 2). This proportion dropped to $5.1 \%$ when adding a fourth behaviour, adherence to recommended daily F\&V intake. Only $2.8 \%$ of the study population adhered to all four behaviours and also had normal body weight (18.49 to $24.99 \mathrm{~kg} / \mathrm{m}^{2}$ ).

\section{Prudence Score}

The mean Prudence Score for the study sample was 5.80 (95\% CI 5.75-5.85), with scores approximating a 'normal' distribution. Less than $1 \%$ reported a Prudence score of ' 0 ', similarly only $2.8 \%$ adhered to five important recommendations: BMI within normal range, sufficient physical activity, within limits alcohol intake, being a non-smoker and sufficient F\&V intake. Women had a significantly higher age-standardized mean Prudence Score than men (5.98 versus 5.41, $\mathrm{t}=10.57 ; \mathrm{df}=4065 ; \mathrm{p}<0.001$ ). Table 3 shows the mean Prudence Score was lower in men than in women for all age-groups, but tended to increase with age in both genders. Patients with tertiary education had higher scores than those with high school education (5.98 versus $5.55 ; \mathrm{t}=8.02, \mathrm{df}=4050, \mathrm{p}<0.001$ ). Regardless of educational background, marital status or employment status, women reported following a healthier lifestyle than men (Table 3).

Table 4 displays the results of a multinomial regression model examining associations between socio-demographic factors and 3 categories of the Prudence Score. After simultaneous adjustment for socioeconomic status and all other factors in the model there was an increased risk of unhealthy diet and lifestyle for males (Relative Risk Ratio $(R R R)=3.03 ; 95 \% \mathrm{CI}=[2.42-3.79])$, younger age $(R R R=$ 3.76 ; $[2.83-5.01])$ and lower educational attainment (RRR $=2.82 ;$ [2.11-3.76]). Of these factors, age between 18 to 39 years and educational attainment below high school were the strongest predictors of unhealthier lifestyle behaviours. As the interaction terms were significant for age and gen$\operatorname{der}\left(\chi^{2}=180.4, \mathrm{df}=10, \mathrm{p}<0.05\right)$ as well as for education and employment status $\left(\chi^{2}=83.0, \mathrm{df}=14, \mathrm{p}<0.05\right)$, the main effects model was adjusted for these potential 


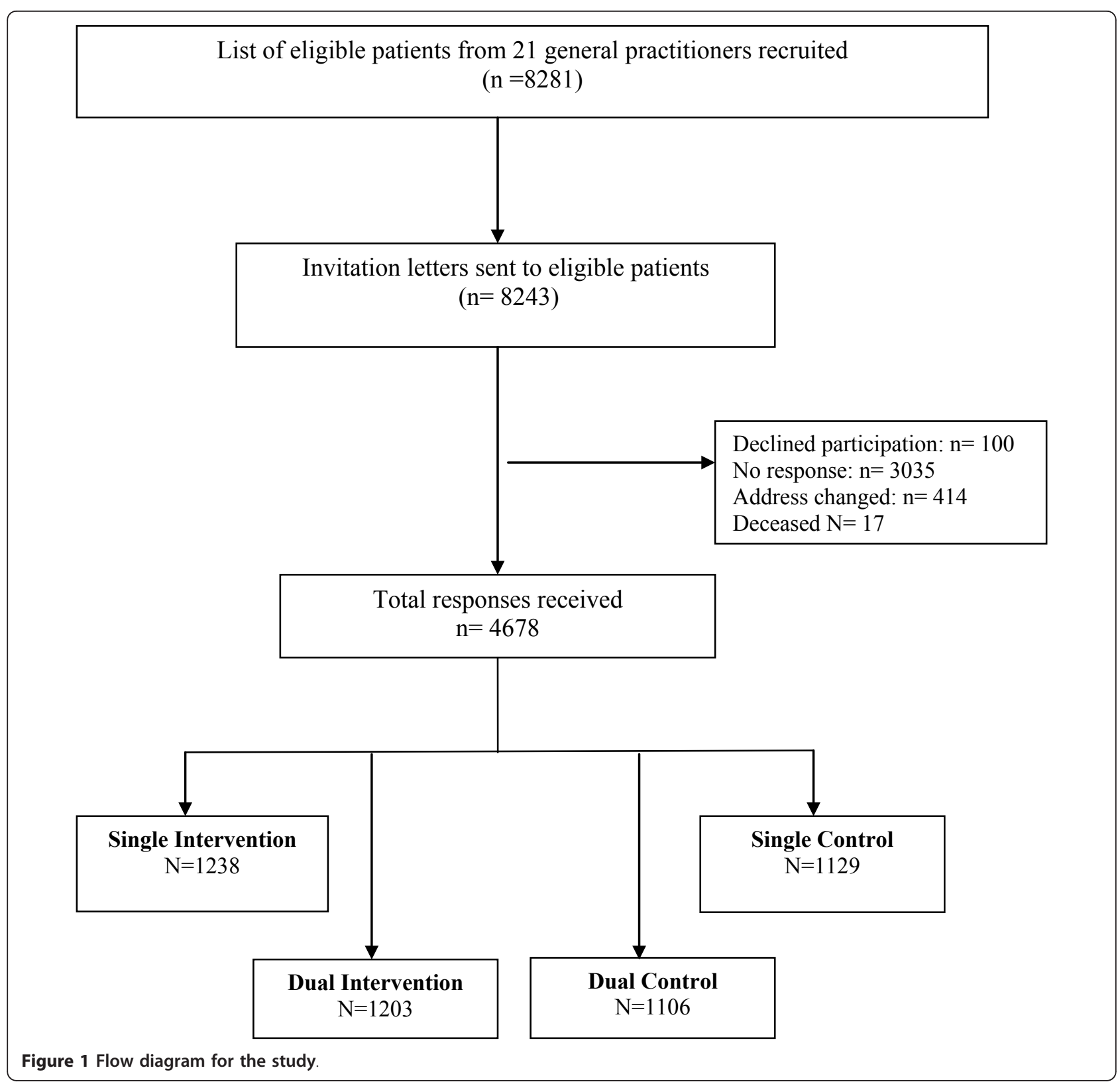

confounders. Following the re-analysis marital status and employment status were no longer significantly associated with Prudence Score.

\section{Discussion}

This paper describes the study procedures for the 10 Small Steps (10SS) study and provides the baseline characteristics of a sample of the 4678 participants who took part in the study. The 10SS approach differs from other studies in its focus by delivering a health behaviour intervention with minimal interruption to general practice routine care and communicating health behaviour advice using a lifestyle score. The Prudence Score showed that despite a relatively high adherence to at least some health behaviours, there was considerable scope for improvement. Only $2.8 \%$ of general practice patients adhered to five important protective behaviours: being a non-smoker, sufficient physical activity, safe alcohol intake, eating 7 or more serves of fruit and vegetables daily, and having a normal BMI. Providing computer-tailored feedback that was endorsed by the patient's GP might have contributed to the participation of a relatively large cohort. This cohort will provide the power to continue with a factorial randomised trial with a good chance of detecting even small changes in behaviour attributable to this minimal intervention. 
Table 1 Socio-demographic and health behaviour characteristics at baseline [mean( $\pm \mathrm{SE}$ ) and number(percentages)]

\begin{tabular}{|c|c|c|c|c|}
\hline CHARACTERISTICS & ALL PERSONS & INTERVENTION & CONTROL & P-VALUE \\
\hline \multicolumn{5}{|l|}{ DEMOGRAPHICS } \\
\hline MEAN AGE & $49.7( \pm 1.1)$ & $49.8( \pm 1.1)$ & $49.7( \pm 1.2)$ & 0.846 \\
\hline GENDER (\% WOMEN) & $3229(68.7 \%)$ & $1688(68.0 \%)$ & $1541(69.5 \%)$ & 0.257 \\
\hline$\overline{\text { MARRIED OR LIVING AS MARRIED }}$ & $3233(69 \%)$ & $1690(68.9 \%)$ & $1543(69.1 \%)$ & 0.470 \\
\hline EDUCATION (\%TERTIARY) & $2660(56.8 \%)$ & $1406(52.9 \%)$ & $1254(56.2 \%)$ & 0.202 \\
\hline \multicolumn{5}{|l|}{ HEALTH BEHAVIOURS } \\
\hline Meat Intake $\leq 4$ Serves Per Week & $3245(69.2 \%)$ & $1689(68.8 \%)$ & $1556(69.5 \%)$ & 0.313 \\
\hline Fish Intake $\geq 2$ Serves Per Week & $3186(67.8 \%)$ & $1666(67.9 \%)$ & $1520(67.8 \%)$ & 0.485 \\
\hline Use Of Low Or No Fat Milk & $3254(69.3 \%)$ & $1688(68.7 \%)$ & $1566(69.9 \%)$ & 0.193 \\
\hline Salt: No Added Salt & $2033(43.4 \%)$ & $1056(43.2 \%)$ & $977(43.6 \%)$ & 0.397 \\
\hline Vegetables And Fruit: 7 Serves Per Day & $579(12.4 \%)$ & $317(13.0 \%)$ & $262(11.7 \%)$ & 0.102 \\
\hline Use Of Spreads Other Than Butter & $3109(66.3 \%)$ & $1639(66.9 \%)$ & $1470(65.6 \%)$ & 0.181 \\
\hline Physical Activity $\geq 150$ Minutes Per Week & $2356(50.3 \%)$ & $1231(50.2 \%)$ & $1125(50.4 \%)$ & 0.469 \\
\hline Alcohol $\leq 2$ Standard Drinks Per Day & $3216(68.4 \%)$ & $1671(68.0 \%)$ & $1545(68.9 \%)$ & 0.271 \\
\hline No Smoking & $4027(86.1 \%)$ & $2105(86.0 \%)$ & $1922(86.3 \%)$ & 0.381 \\
\hline Body weight between 18.5 to $24.99 \mathrm{~kg} / \mathrm{m} 2$ & $1861(41.0 \%)$ & $1003(42.2 \%)$ & $858(39.8 \%)$ & 0.147 \\
\hline
\end{tabular}

T-Test Tested Continuous Variables

Chi-Squared Tested Categorical Variables

The dietary and lifestyle items included in the study are based on epidemiological evidence demonstrating their association with morbidity and mortality [11,29,33-36]. Behaviours such as smoking and physical activity have direct evidence for prevention of NCDs, whereas other behaviours, such as drinking low fat milk and using spreads other than butter, are indirectly supported due to their role in reducing daily saturated fat intake. A previous study by Spencer and Jamrozik et al., using eight of the items used in this study, suggested that in a large cohort of elderly men the combined score had a linear association with all-cause mortality over the subsequent five years and each health behaviour was individually associated with reduced risk of death due to Myocardial Infarction $[13,37]$.
While the protocol developed for this study proved feasible in a clear majority of practices that were approached, the uptake of invitations by patients was incomplete and the predominance of female participants reflected the low 'visibility' of men attending health services, especially in the first half of adult life. Australian data report that female patients account for $57.6 \%$ of General Practice consultations [14]. The consistently higher Prudence Score among older participants and women is notable, and consistent with previous reports that conclude women to be more health conscious than men $[38,39]$. Another explanation could be that females and older participants have more frequent health service contacts, each of which provides an occasion for opportunistic health promotion advice [39] Our data suggest

Table 2 Proportion of the general practice population adhering to recommendations for combined health behaviours

\begin{tabular}{|c|c|c|c|c|c|c|c|}
\hline & \multicolumn{7}{|c|}{$\%$ adherence to health behaviours } \\
\hline & $\mathbf{N}$ & None & One & Two & Three & Four & All Five \\
\hline $\mathrm{Smk}^{\mathrm{a}}$ & 4654 & 13.9 & 86.1 & & & & \\
\hline Smk+Alc ${ }^{b}$ & 4645 & 7.5 & 30.4 & 62.1 & & & \\
\hline $\mathrm{Smk}+\mathrm{Alc}+\mathrm{Pa}^{\mathrm{c}}$ & 4447 & 3.2 & 17.6 & 49.2 & 30.0 & & \\
\hline$\overline{S m k+A l c+P A+V F^{d}}$ & 4437 & 3.0 & 16.8 & 43.7 & 31.3 & 5.1 & \\
\hline Smk+Alc+PA+BMI & 4311 & 1.6 & 11.6 & 36.0 & 36.1 & 14.7 & \\
\hline Smk+Alc+PA+BMI+VF & 4303 & 1.5 & 11.2 & 32.4 & 34.8 & 17.3 & 2.8 \\
\hline
\end{tabular}

a: Non-smoker

b: Alcohol two or less standard drinks per day or a non-drinker

c: Following physical activity guidelines (150 minutes of moderate or 60 minutes of vigorous physical activity per week)

d: Greater than or equal to 7 serves of fruit and vegetables per day

e: Body mass index between $18.49 \mathrm{~kg} / \mathrm{m}^{2}$ and $24.99 \mathrm{~kg} / \mathrm{m}^{2}$ 
Table 3 Distribution of mean Prudence Score by socio-demographic variables and effect of gender

\begin{tabular}{|c|c|c|c|c|c|c|c|c|c|}
\hline & Total & & & Men & & & Women & & \\
\hline & Mean & $95 \% \mathrm{Cl}$ & P-value* & Mean & $95 \% \mathrm{Cl}$ & P-value* & Mean & $95 \% \mathrm{Cl}$ & P-value* \\
\hline All $(N=4281)$ & 5.80 & $5.75-5.85$ & & & & & & & \\
\hline \multicolumn{10}{|l|}{ Gender } \\
\hline Female $(\mathrm{N}=2928)$ & 5.98 & $5.92-6.04$ & & & & & & & \\
\hline Male $(N=1353)$ & 5.41 & $5.32-5.50$ & $<0.001$ & & & & & & \\
\hline \multicolumn{10}{|l|}{ Age Group (Years) } \\
\hline$<50$ years $(N=2170)$ & 5.56 & $5.49-5.63$ & $<0.001$ & 5.00 & $4.87-5.14$ & $<0.001$ & 5.76 & $5.68-5.84$ & $<0.001$ \\
\hline 50 and above $(\mathrm{N}=1893)$ & 6.08 & $6.01-6.15$ & & 5.75 & $5.63-5.87$ & & 6.28 & $6.19-6.36$ & \\
\hline \multicolumn{10}{|l|}{ Education Level } \\
\hline University/Diploma/trade $N=2366$ & 5.98 & $5.91-6.04$ & $<0.001$ & 5.63 & $5.52-5.75$ & $<0.001$ & 6.16 & $6.08-6.23$ & \\
\hline High School and below $\mathrm{N}=1686$ & 5.55 & $5.47-5.63$ & & 5.03 & $4.89-5.17$ & & 5.76 & $5.67-5.86$ & \\
\hline \multicolumn{10}{|l|}{ Marital Status } \\
\hline Married ( $N=2809)$ & 5.84 & $5.78-5.90$ & & 5.49 & $5.39-5.60$ & & 6.03 & $5.95-6.10$ & \\
\hline Other $(\mathrm{N}=1252)$ & 5.71 & $5.62-5.81$ & 0.02 & 5.16 & 4.99-5.33 & 0.001 & 5.91 & $5.80-6.01$ & 0.07 \\
\hline \multicolumn{10}{|l|}{ Employment Status } \\
\hline Employed $(\mathrm{N}=2897)$ & 5.77 & $5.71-5.83$ & & 5.39 & $5.29-5.49$ & & 5.96 & $5.89-6.03$ & \\
\hline Not employed $(\mathrm{N}=1159)$ & 5.88 & $5.79-5.98$ & 0.04 & 5.47 & $5.28-5.67$ & 0.31 & 6.03 & $5.92-6.14$ & 0.33 \\
\hline \multicolumn{10}{|c|}{ Socioeconomic index of economic resources } \\
\hline Advantage $(\mathrm{N}=1987)$ & 5.95 & $5.87-6.02$ & & 5.58 & $5.44-5.71$ & & 6.10 & $6.02-6.18$ & \\
\hline Disadvantaged $(\mathrm{N}=2073)$ & 5.67 & $5.60-5.74$ & $<0.001$ & 5.27 & $5.15-5.39$ & $<0.001$ & 5.87 & $5.79-5.96$ & $<0.001$ \\
\hline
\end{tabular}

*Two sample $T$-test used for assessing the difference between sub-groups.

Table 4 Multivariate associations between Prudence Score and socio-demographic variables

\begin{tabular}{|c|c|c|c|}
\hline \multicolumn{4}{|c|}{ Prudence Score* } \\
\hline & Low Score: High Score & Medium Score: High Score & Test of Significance $^{\#}$ \\
\hline \multicolumn{4}{|l|}{ Gender } \\
\hline Female & 1 & 1 & \\
\hline Male & $3.03(2.42-3.79)$ & $1.69(1.35-2.11)$ & $P<0.001$ \\
\hline \multicolumn{4}{|l|}{ Age group } \\
\hline $60+$ & 1 & 1 & \\
\hline 40 to 59 & $2.32(1.80-2.99)$ & $1.52(1.20-1.93)$ & $P<0.001$ \\
\hline 18 to 39 & $3.76(2.83-5.01)$ & $1.63(1.24-2.15)$ & \\
\hline \multicolumn{4}{|l|}{ Marital Status } \\
\hline Married & 1 & 1 & $P=0.18$ \\
\hline Other & $1.19(0.97-1.47)$ & $1.07(0.87-1.31)$ & \\
\hline \multicolumn{4}{|l|}{ Employment Status } \\
\hline Employed & 1 & 1 & \\
\hline Not Employed & $1.00(0.80-1.25)$ & $0.85(0.69-1.06)$ & $P=0.12$ \\
\hline \multicolumn{4}{|l|}{ Education } \\
\hline University & 1 & 1 & \\
\hline Diploma or trade & $1.47(1.15-1.87)$ & $1.15(0.91-1.45)$ & \\
\hline High school & $1.96(1.50-2.55)$ & $1.31(1.02-1.70)$ & $P<0.001$ \\
\hline Less than high school & $2.82(2.11-3.76)$ & $1.52(1.14-2.01)$ & \\
\hline \multicolumn{4}{|c|}{ Socioeconomic Status index for economic resources } \\
\hline Advantaged & 1 & 1 & \\
\hline Disadvantaged & $0.91(0.71-1.18)$ & $0.96(0.76-1.23)$ & $P=0.22$ \\
\hline Most disadvantaged & $0.81(0.55-1.18)$ & $0.92(0.63-1.34)$ & \\
\hline
\end{tabular}

*Prudence Score defined as low (0 to 5), medium (6 \& 7) and high (8 to 10).

\#Adjusted Wald tests of significance of individual parameters after adjustment for other variables in the model. 
that alternative ways to engage men in health promotion activities need to be sought. For example, the addition of a lifestyle score during a 'Men's Health check' (for Australian men between 45-49 years), might be helpful to increase participation of men in health promotion activities.

The main limitation on this study is the non-response from $40 \%$ of invited patients; however, this response fraction is similar to that of other large community surveys $[40,41]$. Furthermore, the data collected on diet and lifestyle is comparable with other population-wide data for Australia. For example, $86.1 \%$ of participants were nonsmokers (never-and ex-smokers combined) compared with a national figure of $83.4 \%$ [42]. The mean BMI was $26.7 \mathrm{~kg} / \mathrm{m} 2$, with $56.1 \%$ of participants in the 'overweight' or 'obese' range, which is again consistent with a national estimate of 58.5\% [43]. Despite the underrepresentation of males, the study sample may be considered broadly representative of the adult Australian population.

A strength of this protocol is the systematic approach to data collection. The data collection tool is a reliable and valid instrument [23] that can be used in clinical settings to examine and measure multiple risk behaviours associated with chronic conditions. The added value of the Prudence Score is its ease of application without requiring invasive biological measures. It will help to identify patients at higher risk of non-communicable diseases, as well as guide the development of effective strategies for risk-reduction and prevention. The protocol was successfully implemented in order to collect the data and to test the intervention. This study protocol may be useful in general practice for measuring multiple health behaviours simultaneously and communicating the results to patients using the Prudence Score. The next step in the 10SS study is to evaluate the extent to which this minimal computer-tailored intervention leads to positive health behaviour change in the short (three months) and long term (12 months).

\section{Additional material}

Additional file 1: Promoting Healthy Communities.

\begin{abstract}
Abbreviations
10SS: 10 Small steps; NCDs: Non-communicable diseases; BMI: Body mass index; GPs: General practitioners; NHMRC: National health and medical research council; NHF: National heart foundation of Australia; SES: Socioeconomic status.
\end{abstract}

\section{Acknowledgements}

We acknowledge the late Professor Konrad Jamrozik's contribution to the conception, design and implementation of the study prior to his death in March 2009. This study was supported by the MBF Foundation grant, Australia. Dr SP is a National health and Medical Research Council (NHMRC) supported postgraduate scholar. Dr CV is a research fellow, supported by NHMRC and the National Heart Foundation, Australia.

\section{Author details}

${ }^{1}$ School of Population Health, The University of Queensland, Herston Road, Herston, Queensland 4006, Australia. ${ }^{2}$ Healthy Communities Research Centre, The University of Queensland, Salisbury Road, Ipswich, Queensland 4305, Australia. ${ }^{3}$ Central Queensland University, Institute for Health and Social Science Research, Bruce Highway, Rockhampton, Queensland 4700, Australia. ${ }^{4}$ School of Medicine, The University of Queensland, Herston Road, Herston, Queensland 4006, Australia. ${ }^{5}$ Health Systems and Policy Academic Discipline Group, School of Population Health, The University of Queensland, Herston Road, Herston, Queensland 4006, Australia.

\section{Authors' contributions}

SP made a substantial contribution to the concept, design, data collection, data management, data interpretation and drafting the manuscript. CV supervised development of the computer-tailored health promotion advice as well as contributed to the draft. DK contributed to the development of the study, data interpretation and was involved in drafting the manuscript. FB supervised the implementation of the study and was involved in drafting the manuscript. All authors read and approved the final manuscript.

\section{Competing interests}

The authors declare that they have no competing interests.

Received: 13 February 2012 Accepted: 12 March 2012

Published: 12 March 2012

\section{References}

1. Beaglehole R, Ebrahim S, Reddy S, Voute J, Leeder S: Prevention of chronic diseases: a call to action. Lancet 2007, 370(9605):2152-2157.

2. World Health Organisation: Prevention and Control of Noncommunicable Diseases: Implementation of the Global Strategy Geneva: World Health Organisation; 2008

3. Australian Institute of Health and Welfare: Chronic Disease and Associated Risk Factors, 200 Canberra: AlHW; 2002.

4. Dickinson HO, Mason JM, Nicolson DJ, Campbell F, Beyer FR, Cook JV, Williams B, Ford GA: Lifestyle interventions to reduce raised blood pressure: a systematic review of randomised controlled trials. J Hypertens 2006, 24(2):215-233.

5. Kromhout D, Menotti A, Kesteloot $H$, Sans S: Prevention of coronary heart disease by diet and lifestyle: evidence from prospective cross-cultural, cohort, and intervention studies. Circulation 2002, 105(7):893-898.

6. Prochaska JO, Velicer WF, Redding C, Rossi JS, Goldstein M, DePue J, Greene GW, Rossi SR, Sun X, Fava JL, Laforge R, Rakowski W, Plummer BA: Stage-based expert systems to guide a population of primary care patients to quit smoking, eat healthier, prevent skin cancer, and receive regular mammograms. Prev Med 2005, 41(2):406-416.

7. World Health Organisation: Preventing Chronic Diseases: A Vital Investment World Health Organisation Global Report 200 Geneva: WHO; 2005.

8. Begg S, Vos T, Barker B, Stevenson C, Stanley L, Lopez AD: The Burden of Disease and Injury in Australia 2003. PHE 8 Canberra: AlHW; 2007.

9. Key TJ, Allen NE, Spencer EA, Travis RC: The effect of diet on risk of cancer. Lancet 2002, 360(9336):861-868.

10. Kromhout D, de Lezenne Coulander C: Diet, prevalence and 10-year mortality from coronary heart disease in 871 middle-aged men. The Zutphen Study. Am J Epidemiol 1984, 119(5):733-741.

11. Mente A, de Koning L, Shannon HS, Anand SS: A systematic review of the evidence supporting a causal link between dietary factors and coronary heart disease. Arch Intern Med 2009, 169(7):659-669.

12. Myint PK, Luben RN, Wareham NJ, Bingham SA, Khaw KT: Combined effect of health behaviours and risk of first ever stroke in 20,040 men and women over 11 years' follow-up in Norfolk cohort of European Prospective Investigation of Cancer (EPIC Norfolk): prospective population study. Br Med J 2009, 338:b349.

13. Spencer CA, Jamrozik K, Norman PE, Lawrence-Brown M: A simple lifestyle score predicts survival in healthy elderly men. Prev Med 2005, 40(6):712-717.

14. Britt H, Miller GC, Charles J, Henderson J, Bayram C, Valenti L, Pan Y, Harrison C, Fahridin S, O'Halloran J: General Practice Activity in Australia 199900 to 2008-09: 10 Year Data Table Canberra: AlHW; 2010.

15. Glasgow RE, Bull SS, Gillette C, Klesges LM, Dzewaltowski DA: Behaviour change intervention research in healthcare settings: a review of recent 
reports with emphasis on external validity. Am J Prev Med 2002, 23(1):62-69.

16. Sacks FM, Appel L, Moore TJ, Obarzanek E, Vollmer WM, Svetkey LP, Bray GA, Vogt TM, Cutler JA, Windhauser MM, Lin PH, Karanja N: A dietary approach to prevent hypertension: a review of the Dietary Approaches to Stop Hypertension (DASH) Study. Clin Cardiol 1999, 22(7 Suppl):III6-III10.

17. Ashenden R, Silagy C, Weller D: A systematic review of the effectiveness of promoting lifestyle change in general practice. Fam Pract 1997, 14(2):160-176

18. Goldstein MG, Whitlock EP, DePue J: Multiple behavioural risk factor interventions in primary care. Summary of research evidence. Am J Prev Med 2004, 27(2 Suppl):61-79.

19. Harris M, Powell Davies G: SNAP: A journey from research to policy to implementation and back. N S W Public Health Bull 2005, 16(1112):195-199

20. Young JM, Ward JE: Implementing guidelines for smoking cessation advice in Australian general practice: opinions, current practices, readiness to change and perceived barriers. Fam Pract 2001, 18(1):14-20.

21. Orleans $C T$ : Addressing multiple behavioural health risks in primary care. Broadening the focus of health behaviour change research and practice. Am J Prev Med 2004, 27(2 Suppl):1-3.

22. Harris M: The Role of Primary Health Care in Preventing the Onset of Chronic Disease, with a Particular Focus on the Lifestyle Risk Factors of Obesity, Tobacco and Alcohol Sydney: Centre for Primary Health Care and Equity, University of New South Wales; 2008.

23. Parekh S, King D, Owen N, Jamrozik K: Spousal concordance and reliability of the 'Prudence Score' as a summary of diet and lifestyle. Aust N Z J Public Health 2009, 33(4):320-324.

24. Craig CL, Marshall AL, Sjostrom M, Bauman AE, Booth ML, Ainsworth BE, Pratt M, Ekelund U, Yngve A, Sallis JF, Oja P: International physical activity questionnaire: 12-country reliability and validity. Med Sci Sports Exerc 2003, 35(8):1381-1395.

25. National Health and Medical Research Council: Dietary guidelines for Australian adults. 2003 [http://www.nhmrc.gov.au/_files_nhmrc/ publications/attachments/n33.pdf].

26. National Heart Foundation and Cardiac Society of Australia and New Zealand: Lipid Management Guidelines 2001 - Summary Paper. Med J Aust 2001, 175:S57-S88.

27. National Heart Foundation: Salt and hypertension (Professional Paper). PP 555 May 2007. Website consulted 2008 [http://www.heartfoundation.org.au/ SiteCollectionDocuments/salt-and-hypertension.pdf].

28. Haskell WL, Lee IM, Pate RR, Powell KE, Blair SN, Franklin BA: Physical activity and public health: updated recommendation for adults from the American College of Sports Medicine and the American Heart Association. Med Sci Sports Exerc 2007, 39:1423-1434.

29. National Health and Medical Research Council: Australian Guidelines to Reduce Health Risks from Drinking Alcohol Commonwealth of Australia Canberra; 2009.

30. National Heart Foundation: Cigarette smoking. 2002 [http://www heartfoundation.org.au/SiteCollectionDocuments/Cigarette-Smoking.pdf], [cited 2008 Feb 19].

31. National Institutes of Health Expert Committee: Clinical guidelines on the identification, evaluation, and treatment of overweight and obesity in adults-The evidence report. Obes Res 1998, 6(Suppl 2):51S-209S.

32. Australian Bureau of Statistics: 2006 Census of Population and Housing Product Brief. Socio-Economic Indexes for Areas (SEIFA 2006) cat. no. 2033.0.55.001. Australian Breau of Statistics, editor. Canberra; 2006.

33. Tamakoshi A, Tamakoshi K, Lin Y, Yagyu K, Kikuchi S: Healthy lifestyle and preventable death: findings from the Japan Collaborative Cohort (JACC) Study. Prev Med 2009, 48(5):486-492.

34. Summerbell CD, Cameron C, Glasziou PP: Advice on low-fat diets for obesity. Cochrane Database of Systematic Reviews 2008, 3, Art.No.: CD003640. DOI: 10.1002/14651858.CD003640.pub2.

35. Sofi F, Cesari F, Abbate R, Gensini GF, Casini A: Adherence to Mediterranean diet and health status: meta-analysis. BMJ 2008, 337 a1344.

36. He FJ, Macgregor GA: A comprehensive review on salt and health and current experience of worldwide salt reduction programmes. J Hum Hypertens 2008, 23(6):363-84.
37. Spencer CA, Jamrozik K, Lawrence-Brown M, Norman PE: Lifestyle still predicts mortality in older men with established vascular disease. Prev Med 2005, 41(2):583-588.

38. Hjartaker A, Lund E: Relationship between dietary habits, age, lifestyle, and socio-economic status among adult Norwegian women. The Norwegian Women and Cancer Study. Eur J Clin Nutr 1998, 52(8):565-572.

39. Courtenay WH: Constructions of masculinity and their influence on men's well-being: a theory of gender and health. Soc Sci Med 2000, 50(10):1385-1401.

40. Eakin E, Reeves M, Lawler S, Graves N, Oldenburg B, Del Mar C, Wilke K, Winkler E, Barnett A: Telephone counseling for physical activity and diet in primary care patients. Am J Prev Med 2009, 36(2):142-149.

41. van Sluijs EM, van Poppel MN, Twisk JW, Chin APMJ, Calfas KJ, van Mechelen W: Effect of a tailored physical activity intervention delivered in general practice settings: results of a randomised controlled trial. Am J Public Health 2005, 95(10):1825-1831.

42. Australian Institute of Health and Welfare:: Australia's Health 2008. Cat. no. GEP 22. Australian Institute of Health and Welfare: Australia's Health 2008. Canberra AlHW; 2008.

43. Britt H, Miller GC, Charles J, et al: General practice activity in Australia 2007-08. Cat. no. GEP 22. Australian Institute of Health and Welfare;

\section{Pre-publication history}

The pre-publication history for this paper can be accessed here: http://www.biomedcentral.com/1471-2458/12/179/prepub

\section{doi:10.1186/1471-2458-12-179}

Cite this article as: Parekh et al:: Design and baseline characteristics of the 10 Small Steps Study: a randomised controlled trial of an intervention to promote healthy behaviour using a lifestyle score and personalised feedback. BMC Public Health 2012 12:179.

\section{Submit your next manuscript to BioMed Central and take full advantage of:}

- Convenient online submission

- Thorough peer review

- No space constraints or color figure charges

- Immediate publication on acceptance

- Inclusion in PubMed, CAS, Scopus and Google Scholar

- Research which is freely available for redistribution

Submit your manuscript at www.biomedcentral.com/submit
Biomed Central 